\title{
Studies on bulk growth, structural and microstructural characterization of 4-aminobenzophenone single crystal grown from vertical Bridgman technique
}

\author{
SP PRABHAKARAN ${ }^{1}$, R RAMESH BABU $^{1, *}$, G BHAGAVANNARAYANA $^{2}$ and K RAMAMURTHI ${ }^{3}$ \\ ${ }^{1}$ Department of Physics, Bharathidasan University, Tiruchirappalli 620 024, Tamil Nadu, India \\ ${ }^{2}$ X-Ray and Crystal Growth Section, National Physical Laboratory, New Delhi 110 012, India \\ ${ }^{3}$ Department of Physics and Nanotechnology, Faculty of Engineering and Technology, SRM University, Kattankulathur \\ 603 203, India
}

MS received 29 February 2012; revised 1 April 2013

\begin{abstract}
Bulk single crystal of 4-aminobenzophenone with a size of $25 \mathrm{~mm}$ dia. and $35 \mathrm{~mm}$ length has been grown by vertical Bridgman technique. The crystal system of the grown crystal was confirmed by X-ray diffraction analysis. Crystalline perfection was analysed by high resolution X-ray diffraction studies. Chemical etching was carried out for the first time in 4-aminobenzophenone single crystal to study the defects presented in the grown crystal and the growth mechanism involved. Several organic etchants were employed with different etching time to select suitable etchant for studying dislocation pattern and other structural defects existing in the grown crystal. Etch patterns such as spirals and striations observed for the selective etchants provide considerable information on growth mechanism of the crystal.
\end{abstract}

Keywords. Organic compound; crystal growth; surface etching; dislocations.

\section{Introduction}

Studies on the nature and distribution of defects in crystals are important because they have deleterious effects on the performance of electronic devices and play an important role in the process of crystal growth. During the last three decades, a variety of methods have been developed for the detection of defects in single crystals (Mendez et al 1995; Huang et al 2005; Wei et al 2008) due to increasing demands on crystals with better perfection for the fabrication of electronic devices. Chemical etching (Prabhakaran et al 1999; Ganesamoorthy et al 2004) is one among them, employed to study different types of etch pits and their density for obtaining useful information on the nature of defects, impurity contents and history of growth mechanism of the crystals. Also, organic materials are in increasing demand, as they are promising candidates for nonlinear optical (NLO) applications (Hierle et al 1984; Driscoll et al 1986; Gunter et al 1987). Particularly, materials with high molecular hyperpolarizabilities have attained greater importance because of their high nonlinear optical coefficients. The higher order polarizability of organic materials is due to the structure which is based on the extended $\pi$-bond system of the molecule. Also, it can be easily manipulated by suitable substitution of electron donating and electron withdrawing groups around the aromatic moieties. In view of

\footnotetext{
*Author for correspondence (rampap2k@yahoo.co.in)
}

this, a number of new organic NLO materials were reported by many researchers (Vijayan et al 2005; Haja Hameed et al 2006). Among them, 4-aminobenzophenone (4-ABP) was first reported by Frazier et al in 1987. It belongs to the monoclinic crystal system with space group $P 2_{1}, Z=2$, $a=12.036 \AA, b=5.450 \AA, c=8.299 \AA$ and $\beta=97.86^{\circ}$ (Genbo et al 1992). Useful optical transmission range of 4-ABP extends from 420 to $1400 \mathrm{~nm}$ and its powder SHG efficiency is 360 times that of ADP (Pan 1994).

As the applications of 4-ABP ( $\mathrm{Li}$ et al 1997a, b) inspire technical innovations, growth of bulk single crystals of 4-ABP has become essential task for the researchers. Generally most of the nonlinear optical organic single crystals can be grown from solution and melt growth techniques (Zhang et al 2009; Arulchakkaravarthi et al 2002). But, growth of 4-ABP is difficult from both solution and melt growth techniques. This is due to that 4-ABP is readily photolysed in solution and changed to radical species which are themselves very reactive. However, reports are available on the growth and studies of 4-ABP single crystals from solution and melt growth techniques (Pan et al 1993, 1997; Lal et al 1997; Li et al 1997a, b; Anandha Babu et al 2009). The reports (Lal et al 1997; Anandha Babu et al 2009) concluded that the melt grown 4-ABP crystals are poor in quality and also small in size. Further, systematic studies are not available on the growth mechanism and for the defects present in the grown 4-ABP crystal. Therefore, in the present investigation, growth of bulk single crystal of 4-aminobenzophenone from vertical 
Bridgman technique is studied. The induced structural defects in terms of growth mechanism are analysed for the grown crystal by chemical etching technique using different etchants at room temperature on the plane $\left(\begin{array}{ll}3 & 2\end{array}\right)$ which is perpendicular to the growth direction. The patterns produced by different organic etchants for different etching time are discussed.

\section{Experimental}

\subsection{Furnace and ampoule design}

The vertical Bridgman system used for the growth of 4ABP single crystal consists of furnace with temperature controller, translation assembly and glass ampoule. In order to overcome thermal instability, low thermal conductivity and high supercooling tendencies of 4-ABP, special efforts have been made on construction of furnace, translation set up and ampoule design. Furnace employed in this work was made up of cylindrical glass tube of $70 \mathrm{~mm}$ dia wound with A1 super kanthal wire of $1 \mathrm{~mm}$ thickness. The furnace temperature was controlled by the temperature controller with an accuracy of $0.1^{\circ} \mathrm{C}$. The winding arrangement in the furnace was made as closer in the middle region gradually from the top and wider in the bottom region. Temperature distribution achieved from this winding arrangement can be divided into three zones such as hotter zone, gradient or growth zone and cooling zone. The axial temperature profile of the furnace used for the growth of 4-ABP is shown in figure 1. To avoid thermal loss and to achieve uniform distribution of heat, the furnace was completely insulated with ceramic wool and an outer cover which is the glass tube of $140 \mathrm{~mm}$ dia. A window $(20 \times 200 \mathrm{~mm})$ was provided through the ceramic wool for monitoring growth at different growth conditions.

Glass ampoules of $1 \mathrm{~mm}$ thick and $25 \mathrm{~mm}$ diameter were used for the growth of bulk single crystal of 4-ABP from

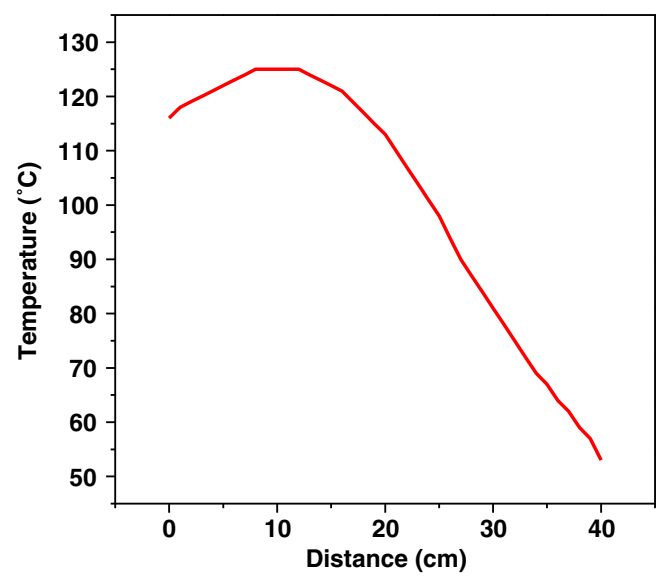

Figure 1. Temperature profile of furnace used for growth of 4-ABP single crystal. vertical Bridgman technique. In order to prevent the generation of defects due to the presence of impurities, the ampoules were cleaned with water and acetone and dried well in a hot air oven. The shape of the ampoule which was used to grow good quality single crystal of 4-ABP in the present work is shown in figure $2 \mathrm{a}$.

\subsection{Growth of 4-ABP single crystal}

The commercially available 4-ABP was purified several times by recrystalization processes and taken for growth in the ampoule. The material filled ampoule was kept at the hotter zone of the furnace and heated to the melting point $\left(121{ }^{\circ} \mathrm{C}\right)$ of the material. The complete melt was kept for 3 to $4 \mathrm{~h}$ to attain thermal equilibrium and was then translated slowly inside the furnace in order to achieve crystallization temperature of the material. Growth was achieved in the growth region by establishing a temperature gradient of $2{ }^{\circ} \mathrm{C} / \mathrm{cm}$. Generally, organic material requires low translation rate at lower temperature region of the furnace. This is mainly due to poor thermal conductivity of the materials which usually take longer time to solidify from the melt as the heat of fusion must be removed through the growing crystal. Hence, different translation rates were attempted for the growth of single crystal of 4-ABP.

For ampoule with $25 \mathrm{~mm}$ diameter, the temperature gradient of $2{ }^{\circ} \mathrm{C} / \mathrm{cm}$ and the translation rate of $0.5 \mathrm{~mm} / \mathrm{h}$ which yielded relatively transparent 4-ABP single crystal are
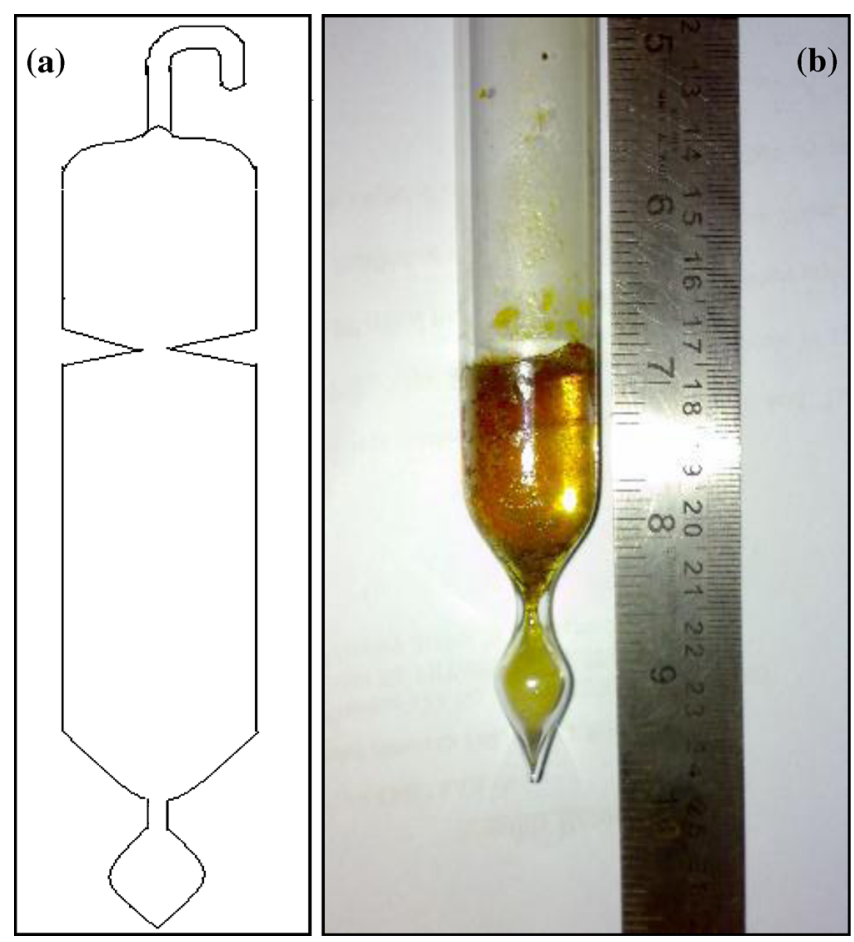

Figure 2. (a) Optimized ampoule shape used for growth of 4ABP single crystal by vertical Bridgman technique and (b) grown 4-ABP crystal with ampoule. 

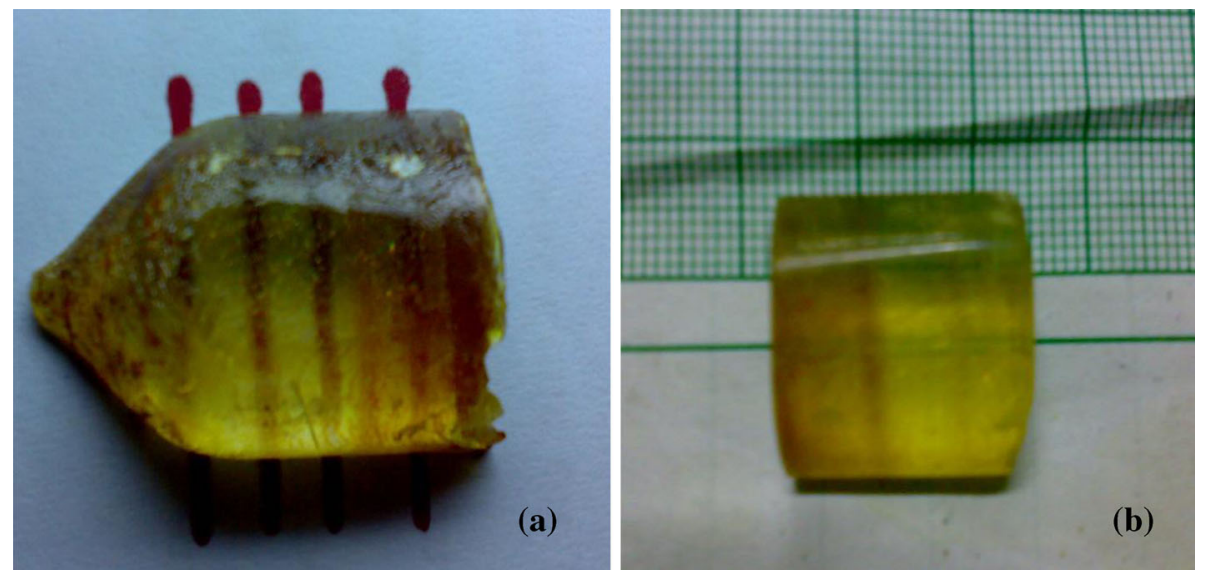

Figure 3. (a) as grown and (b) polished 4-ABP single crystal.

Table 1. Lattice parameter values of 4-aminobenzophenone single crystal.

\begin{tabular}{lccc}
\hline Lattice parameters & Reported $^{*}$ & Present work & Difference \\
\hline$a(\AA)$ & 12.036 & 12.030 & $(-) 0.006$ \\
$b(\AA)$ & 5.450 & 5.476 & $(+) 0.026$ \\
$c(\AA)$ & 8.299 & 8.302 & $(+) 0.003$ \\
$\beta^{\circ}$ & 97.86 & 97.84 & $(-) 0.020$ \\
\hline
\end{tabular}

*Genbo et al (1992)

the optimized growth parameters. Figure $2 \mathrm{~b}$ shows grown 4-ABP crystal with ampoule. The bulb shape at the bottom of ampoule isolates the chemical impurities and multinucleation created at the initial stage. Figure $3 \mathrm{a}$ shows harvested 4 -ABP single crystal from the ampoule. The dimension of the 4-ABP crystal grown from the present experimental set up is $25 \mathrm{~mm}$ dia. and $35 \mathrm{~mm}$ length. Figure $3 \mathrm{~b}$ shows polished 4-ABP crystal.

\subsection{Characterization}

The powder X-ray diffraction pattern of the grown crystal was recorded using XPERT PRO X-ray diffractometer and its lattice parameters were calculated. The structural perfection of the grown single crystal was analysed by high resolution X-ray diffraction (HRXRD) employing a multicrystal X-ray diffractometer developed at NPL (Lal and Bhagavannarayana 1989). The well-collimated and monochromated $\mathrm{MoK} \alpha_{1}$ beam, obtained from the three monochromator Si crystals set in dispersive (+,-,-) configuration, has been used as the exploring X-ray beam. For the chemical etching study, crystal wafers of $5 \mathrm{~mm}$ thickness were cut from the as grown crystal by wet thread method along the growth plane ( $\left.\begin{array}{lll}3 & 2 & 1\end{array}\right)$. Etching was carried out by dipping the cut crystal plates in etchants for the required period at room temperature $\left(28{ }^{\circ} \mathrm{C}\right)$ and then the crystals were wiped with dry filter paper. Toluene, ethanol and acetone were used as the etchants in the present work. Etching was done with different time intervals and the observed patterns were photographed under optical microscope.

\section{Results and discussion}

\subsection{X-ray diffraction}

From the powder X-ray diffraction pattern, the $d$-spacing was calculated and $h k l$ values for the diffraction peaks were indexed. The lattice parameter values of 4-ABP were calculated from the powder XRD pattern and compared with the reported values (Genbo et al 1992) in table 1. It is observed that the numerically calculated lattice parameter values of the grown sample show very slight difference with the reported values which may practically be ignored. Thus the observed values are in good agreement with the reported values and confirm monoclinic crystal system of the grown 4-ABP. Orientation of the growth plane was identified as (32 1) using Xray diffraction analysis of crystal plate cut along the growth direction.

\subsection{HRXRD analysis of ABP single crystal}

Figure 4 shows high-resolution rocking/diffraction curve (DC) recorded for a typical 4-ABP crystal grown by vertical Bridgman method using (3 221 ) diffracting planes in symmetrical Bragg geometry by employing the multicrystal 
X-ray diffractometer with $\mathrm{MoK} \alpha_{1}$ radiation. As seen in the figure, DC contains a single but broad peak having 1350 arc s $(=22.5 \mathrm{arc} \min )$ of full width at half maximum (FWHM). This indicates that the specimen contains not only good number of dislocations, though it does not indicate any major structural grain boundaries, but also mosaic blocks misoriented to each other by few arc min. Thermal strain introduced due to the variation of temperature gradient during growth could be responsible for the observed dislocations. Sometimes thermal strain can also be enhanced by the difference in thermal expansion of the ampoule and of the crystal. It is reflected within the structures formed, as an assemblage of atoms which are made relatively immobile by releasing the energy known as the heat of crystallization. The resulting crystal lattices resemble a mosaic of slightly misaligned adjacent regions.

\subsection{Etching studies}

In single crystals, changes in surface morphology strongly reflect supersaturation, presence of impurities, concentration of impurities in growth medium and temperature used for the growth. The process of chemical etching is able to develop

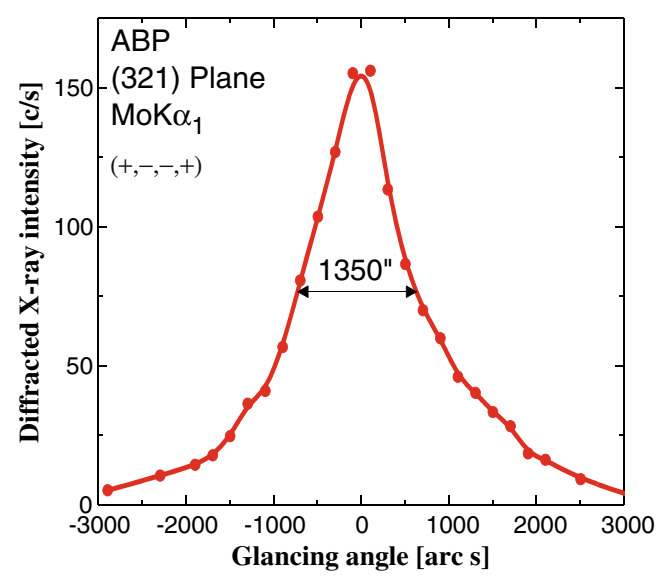

Figure 4. High-resolution X-ray diffraction curve recorded for ABP single crystal. the features such as etch spirals, terraced, flat bottomed pits, etch hillocks, etc on a crystal surface. These patterns are the result of chemical attack at the strain field surrounding the dislocation line. In the present study, interesting results have been observed from the etching of 4-ABP with different organic etchants. Table 2 summarizes the observations of etching behaviour of different etchants on the (32 1) surface of the grown crystal for different etching time. The grown 4$\mathrm{ABP}$ crystal has some incomplete cleavage planes due to the difference in atomic bond strength between the crystal planes during growth. Figure 5a represents presence of such cleavage planes in the as grown 4-ABP single crystal in the direction parallel to the growth. The slow growth rate and cooling rate were used to prevent further cracks induced by the existence of cleavage planes.

3.3a Toluene as etchant: The surface of the as grown (3 21 ) crystal plane is shown in figure $5 \mathrm{~b}$. When this face was etched with toluene for $10 \mathrm{~s}$, it revealed elongated rectangular pattern (macrosteps) having elongation perpendicular to the growth direction with steep slopes parallel to this direction (figure 5c). These macrosteps are the evidence for layer by layer growth mechanism of 4-ABP single crystal. Generally, layer growth requires high degree of uniform supersaturation. But, the non-uniform distribution of the macrosteps on the surface represents non-uniformity of the supersaturation caused by concentration gradient during the growth process (Dhanraj et al 1991). Figure 5d reveals growth sector boundaries formed between the regions of two different orientations. The variation in growth rates of different growth faces resulted in the structure mismatch and lattice distortion between the faces and thus formed boundaries (Wu et al 2005). To avoid the existence of this kind of boundaries, axial and radial temperature gradient should be kept stable during growth. While etching the same face with $20 \mathrm{~s}$ in toluene the etch pit morphology was changed from rectangular pattern to triangular pattern like a valley (figure 5e). Further prolonged etching ( $30 \mathrm{~s}$ ) retained the shape of etch patterns, but became more prominent. It confirms the dislocation sites which dominate the crystal growth, and the formation of valley may be due to the meeting of the two individual dislocation sources.

Table 2. Etching behaviour of different organic etchants on (3 2 1) surface of grown 4-ABP single crystal for different etching time.

\begin{tabular}{lclc}
\hline Etchant & Etching time & Etching characteristics & Fig. No. \\
\hline Toluene & $10 \mathrm{~s}$ & Elongated rectangular patterns & $5 \mathrm{c}$ \\
Toluene & $20 \mathrm{~s}$ & Triangular pits & $5 \mathrm{e}$ \\
Toluene & $30 \mathrm{~s}$ & Triangular pits & $5 \mathrm{f}$ \\
Ethanol & $5 \mathrm{~s}$ & Trapezoid etch pits & $6 \mathrm{a}$ \\
Ethanol & $10 \mathrm{~s}$ & Kinks & $6 \mathrm{~b}$ \\
Acetone & $10 \mathrm{~s}$ & Stacking of planes & $6 \mathrm{c}$ \\
Acetone & $15 \mathrm{~s}$ & Growth spirals & $6 \mathrm{~d}$ \\
\hline
\end{tabular}



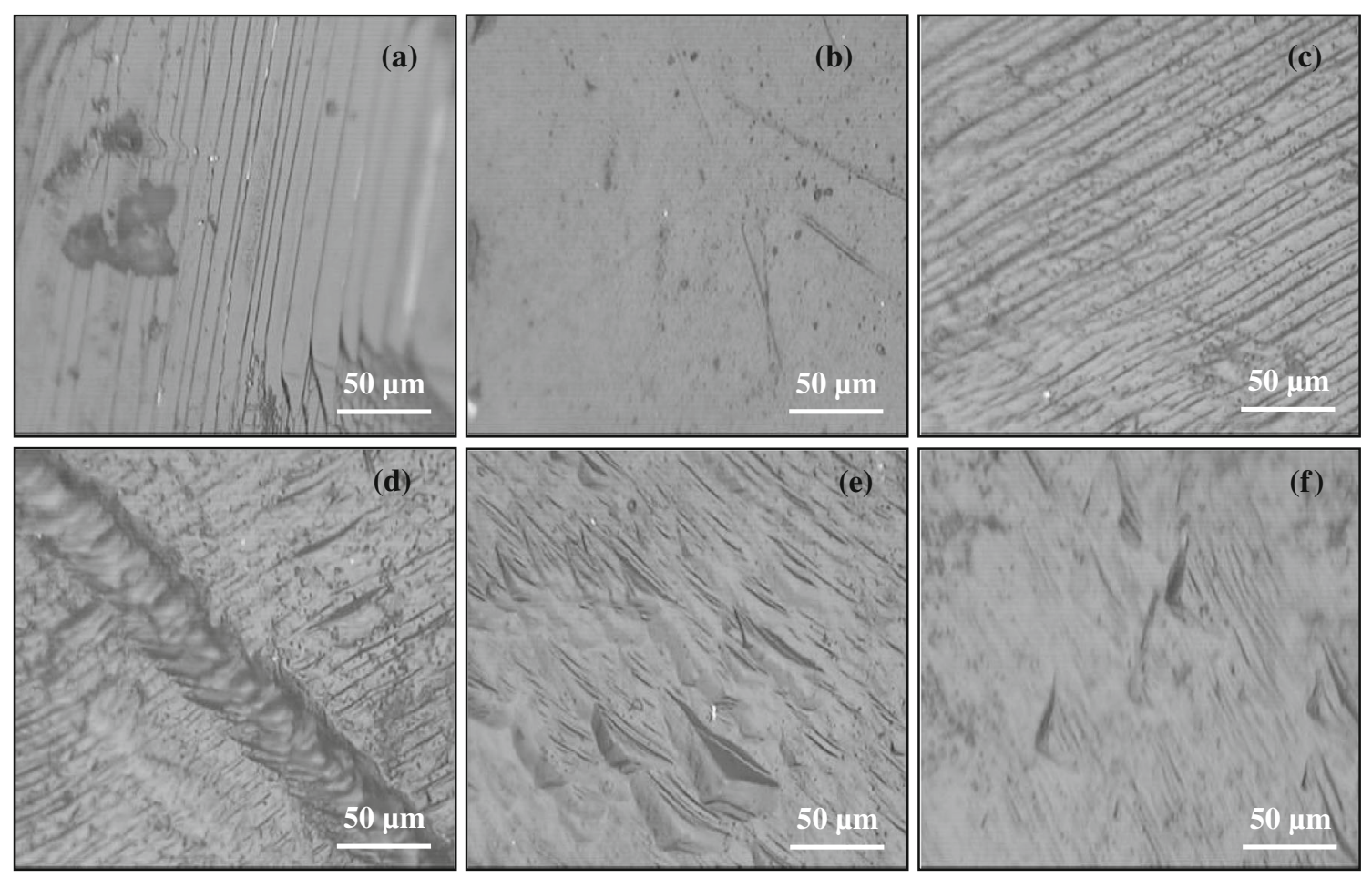

Figure 5. Patterns observed on 4-ABP single crystal: (a) cleavage planes, (b) as grown (3 2 1) plane, (c) and (d) etching with toluene for $10 \mathrm{~s}$, (e) for $20 \mathrm{~s}$, (f) for $30 \mathrm{~s}$.
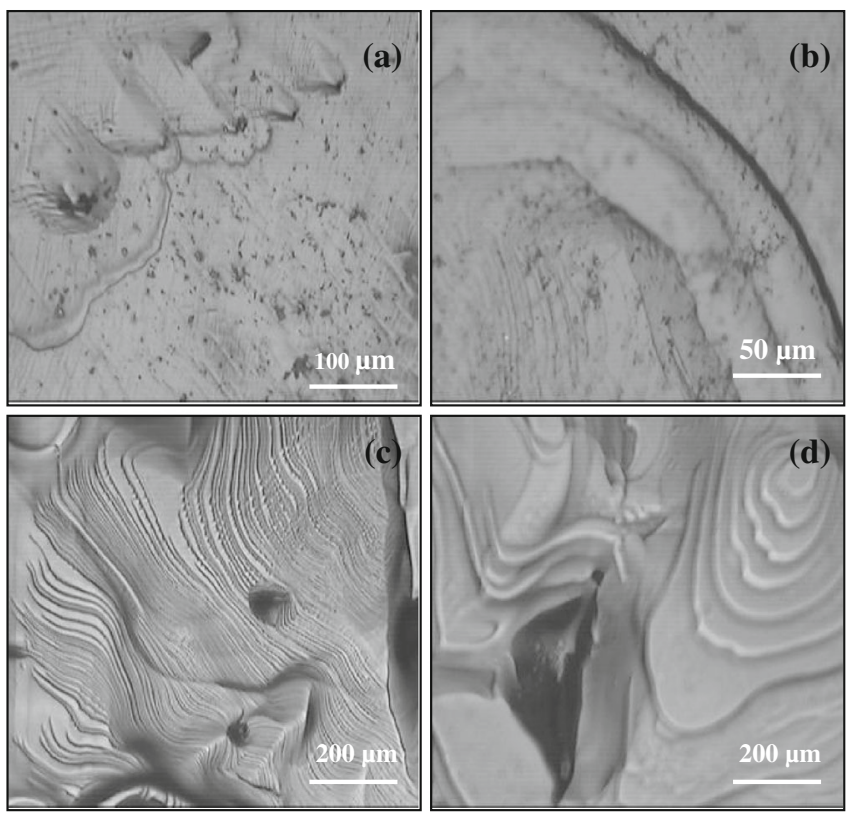

Figure 6. Etch patterns observed for 4-ABP single crystal with ethanol for (a) $5 \mathrm{~s}$, (b) $10 \mathrm{~s}$ and with acetone for (c) $10 \mathrm{~s}$ (d) $15 \mathrm{~s}$.

3.3b Ethanol as etchant: Trapezium shaped etch patterns of asymmetrical form were produced (figure 6a) when the crystal surface was etched with ethanol for $5 \mathrm{~s}$. The asymmetrical etch patterns are the effect of change in supersaturation as a result of thermal fluctuation (Rashkovich et al 1991).
When the same face was etched for $10 \mathrm{~s}$, the kinks were observed as shown in figure $6 \mathrm{~b}$. These may be due to change in diffusion field around the dislocation site with increase of etching time. Ethanol produces no distinct etch pits on prolonged etching. Ethanol, being a solvent of higher dielectric constant among the selected three etchants, the etch pit formation is poor in this case. Hence, it is observed that ethanol is not suitable for etching study, but addition of an acid with ethanol may give well formed etch pits (Sangwal and Szurgot 1982).

3.3c Acetone as etchant: Since 4-ABP is highly soluble in acetone (Lal et al 1997), etching with acetone resulted in complete dissolution of the crystal and no etch pits were observed. The dissolution patterns are shown in figure $6 \mathrm{c}$ and $\mathrm{d}$. For the etching time of $10 \mathrm{~s}$, the existence of steps (figure 6c) on the crystal surfaces enables the detection of the point of emergence of screw dislocation. The prolonged etching brings out growth spirals (figure 6d) as a result of spiral ledge formed by screw dislocation caused by the growth at low supersaturation. Generally, the part around a screw dislocation is not a stack of atomic planes one above the other, but it is rather a single atomic plane in the form of a helicoid or spiral staircase. The part near the dislocation moves with greater angular velocity than the other parts that are farther away and thus a spiral hill is formed (Sangwal 1987). 


\section{Conclusions}

Bulk single crystal of 4-ABP was grown from vertical Bridgman technique. The various growth parameters such as furnace geometry, crucible design, temperature gradient and growth rate were studied. HRXRD analysis indicates that the grown crystal has major dislocations and misoriented mosaic blocks. The microstructure study by chemical etching agrees well with HRXRD analysis and confirms the existence of dislocations in the grown crystal. It is observed from the etch patterns that the growth mechanism of the grown crystal is two dimensional layer by layer growth. Being a poor etchant, ethanol produced no distinct etch pits on prolonged etching. The dissolution patterns obtained from acetone represent screw dislocation due to the result of growth at very low supersaturation.

\section{Acknowledgements}

This research work is financially supported by the Department of Science and Technology, New Delhi (Sanction No: SR/FTP/PS-41/2007). The authors are thankful to DST FIST, Government of India, for providing experimental facility. Authors are thankful to Dr N Kumaran, Department of Metallurgical and Materials Engineering, National Institute of Technology, Tiruchirappalli, for providing facilities to carry out etching studies.

\section{References}

Anandha Babu G, Thirupugalmani K, Jayaprakasan M and Ramasamy P 2009 J. Cryst. Growth 3111607

Arulchakkaravarthi A, Jayavel P, Santhanaraghavan P and Ramasamy P 2002 J. Cryst. Growth 234159

Dhanraj G, Shripati T and Bhat H L 1991 J. Cryst. Growth 113456

Driscoll T A, Hoffman H J, Stone R E and Perkins P E 1986 J. Opt. Soc. Am. B3 683
Frazier C C, Cockerharm M P, Chauchard M A and Lee C H 1987 J. Opt. Soc. Am. B4 1899

Ganesamoorthy S, Bhaumik I, Karnal A K and Wadhawan V K 2004 J. Cryst. Growth 264320

Genbo S, Zhengdong L, Fan P and Youping H 1992 Cryst. Res. Technol. 27587

Gunter P, Bosshard Ch, Sutter K, Arend H, Chapuis G, Tweig R J and Dobrowolski D 1987 Appl. Phys. Lett. 50486

Haja Hameed A S, Rohain S, Yu W C, Tai C Y and Lan C W 2006 J. Cryst. Growth 297146

Hierle R, Badam J and Zyss J 1984 J. Cryst. Growth 69545

Huang W, Wang W, Wang M and Yuan Q 2005 J. Cryst. Growth 274518

Lal K and Bhagavannarayana G 1989 J. Appl. Cryst. 22209

Lal R B, Zhang H W, Wang W S, Agarwal M D and Penn B G 1997 J. Cryst. Growth 174393

Li Z, Wu B and Su G 1997a Appl. Phys. Lett. 70562

Li Z, Wu B, Su G and Gongfan H 1997b J. Cryst. Growth 171 506

Mendez B, Dutta P S, Piqueras J and Dieguez E 1995 Appl. Phys. Lett. 672648

Pan F 1994 Design, growth, perfection, and properties of organic optical crystals for blue light, Ph.D. Dissertation, University of Strathclyde, Glasgow, Scotland

Pan S, Okano Y, Tsunekwa S and Fukuda T 1993 J. Cryst. Growth 129365

Pan F, Sherwood J N and Simpson G S 1997 J. Mater. Chem. 7 1383

Prabhakaran D, Subramanian C, Balakumar S and Ramasamy P 1999 Physica C 31999

Rashkovich L N, Sheknouv and Yu B 1991 J. Cryst. Growth 112 183

Sangwal K 1987 Etching of Crystals, Theory, Experiment and Application. Amsterdam: The Elsevier Science Publisher

Sangwal K and Szurgot M 1982 Cryst. Res. Technol. 1749

Vijayan N, Balamurugan N, Ramesh Babu R, Gopalakrishnan R and Ramasamy P 2005 J. Cryst. Growth 275 e1895

Wei X, Zhao Y, Dong Z and Li J 2008 J. Cryst. Growth 310639

Wu X, Xu J and Jin W 2005 Mater. Charact. 55143

Zhang P, Deng J, Zeng X, Liu Z, Qiu Y, Zhong H, Fan Y, Huang J, Zhang J and Xu K 2009 J. Cryst. Growth 3114708 